\title{
Acupuncture Versus Non-Steroidal Anti-Inflammatory Drugs for Treatment of Chondromalacia Patellae: A Systematic Review and Meta-Analysis of Randomized Controlled Trials
}

\author{
Zheng-Tao Lva Zhi-Qiang Li ${ }^{b} \quad$ Xiang Zhou $^{\mathrm{c}} \quad$ Wen-Wen Mad Jin-Ming Zhang ${ }^{\mathrm{a}}$ \\ An-Min Chen ${ }^{a}$ \\ a Department of Orthopedics, Tongji Hospital, Tongji Medical College, Huazhong University of Science and Technology, Wuhan, China; \\ ${ }^{b}$ Department of Orthopedics, The 457th hospital of People's Liberation Army, Wuhan, China; \\ ${ }^{\mathrm{c}}$ Department of Internal Medicine V, University Hospital Heidelberg, Heidelberg, Germany; \\ d Institute of Integrated Traditional Chinese and Western Medicine, Tongji Hospital, Tongji Medical College, \\ Huazhong University of Science and Technology, Wuhan, China
}

\section{Keywords}

Chondromalacia patellae $\cdot$ Acupuncture $\cdot$ Meta-analysis . Rehabilitation

\section{Summary}

This systematic review aims to evaluate the efficacy and safety of acupuncture therapy for treating patients with chondromalacia patellae compared to non-steroidal antiinflammatory drugs (NSAID). Eight databases including PubMed, EMBASE, CENTRAL, ISI Web of Science, CNKI, Wanfang, VIP, and CBM were searched up to June 2016. Randomized controlled trials (RCT) assessing the efficacy of acupuncture therapy were identified. The patients in experimental groups had to be treated with acupuncture therapy, and the patients in control groups had to be treated with NSAID. The outcome measures included clinical efficacy rates or pain scores. A total of 7 studies met our inclusion criteria. We analyzed the data from these 7 RCT involving 707 participants. All included studies were judged to be at high risk for bias. Compared with NSAID, acupuncture could further improve the clinical efficacy rates (Risk Ratio 2.57; $95 \%$ confidence interval (Cl) $2.02,3.27$ ) and pain scores (Mean Difference $-1.49 ; 95 \% \mathrm{Cl}-2.37,-0.62$ ). No study reported adverse events associated with acupuncture therapy. Based on the findings in the present review, acupuncture therapy is more effective than NSAID in improving clinical efficacy rates and pain scores. However, these results should be interpreted cautiously due to the poor overall methodological quality of the included trials. Additional studies with large-scale and rigorous experimental design are required.

(C) 2016 S. Karger GmbH, Freiburg

\section{Schlüsselwörter}

Chondropathia patellae - Akupunktur - Meta-Analyse . Rehabilitation

\section{Zusammenfassung}

Die vorliegende systematische Übersichtsarbeit verfolgt das Ziel, die Wirksamkeit und Sicherheit von Akupunktur in der Behandlung von Patienten mit Chondropathia patellae im Vergleich zu nichtsteroidalen antiinflammatorischen Arzneimitteln (non-steroidal anti-inflammatory drugs; NSAID) zu evaluieren. Hierzu wurden die 8 Datenbanken PubMed, EMBASE, CENTRAL, ISI Web of Science, CNKI, Wanfang, VIP und CBM auf Einträge bis Juni 2016 durchsucht und randomisierte kontrollierte Studien (randomized controlled trials; RCT) herausgefiltert. Die Patienten in den Versuchsgruppen mussten mit Akupunktur, die Patienten in den Kontrollgruppen mit NSAID behandelt worden sein. Die Verlaufsmessungen schlossen die klinische Wirksamkeit oder Schmerz-Scores ein. Insgesamt erfüllten 7 Studien unsere Einschlusskriterien. Wir analysierten die Daten dieser 7 Studien, die 707 Teilnehmer beinhalteten. Alle eingeschlossenen Studien wurden als mit einem hohen Bias-Risiko behaftet eingestuft. Gegenüber NSAID zeigten sich bei Akupunktur verbesserte klinische Wirksamkeitsraten (Risk Ratio 2,57; 95\% Konfidenzintervall (KI) 2,02; 3,27) und Schmerz-Scores (mittlere Differenz-1,49; 95\%-KI -2,37;-0,62). In keiner der Studien wurden unerwünschte Ereignisse bezüglich der Akupunkturbehandlung berichtet. Laut den Ergebnissen dieser Übersichtsarbeit ist Akupunktur wirksamer hinsichtlich der Verbesserung von klinischen Wirksamkeitsraten und Schmerz-Scores als NSAID. Aufgrund der insgesamt geringen methodologischen Qualität der hier untersuchten Studien sollten diese Ergebnisse allerdings vorsichtig interpretiert werden. Weitere Studien mit groß angelegtem und streng experimentellem Design sind notwendig.

\section{KARGER}

An-Min Chen 


\section{Introduction}

Chondromalacia patellae (CMP), meaning softening of the articular cartilage of the patella, is often accompanied by fibrillation, fissuring, and erosion of the cartilage [1]. It is always associated with the clinical syndrome of retropatellar pain and other symptoms, such as joint swelling, giving way, patellofemoral crepitus, and wasting of the quadriceps [2]. The etiology of CMP is still unclear, but the process is thought to be caused by a trauma to superficial chondrocytes resulting in a proteolytic enzymic breakdown of the matrix [3]. The incidence in the general population is reported to be $25 \%$, and in the athletic population this proportion is even higher [4]. The patients affected by CMP are young, they are often disabled by severe pain behind the patella, and their pain is likely exacerbated by intense physical activity, inclined walking, ascending or descending stairs, and prolonged sitting with flexed knees.

The patellofemoral pain can only be effectively managed if the physicians and practitioners have a good understanding of the biomechanics of patellofemoral joint. Under normal circumstances, the patella is aligned and remains in the trochlear notch of the femur. The divergent quadriceps muscle is linked by the patella to a common tendon, thereby increasing the lever arm of the quadriceps. Factors affecting patellar alignment alter the functional mechanics of the patellofemoral joint, thus predisposing an individual to retropatellar pain. The experience of pain is aggravated due to the increased reaction force on the patellofemoral joint. Parameters such as increased $\mathrm{Q}$ angle, vastus medialis obliquus (VMO) insufficiency, excessive pronation of the patella, and patella alta are involved in the misalignment of the patella, which makes the patella more likely to be wasted [5].

Theoretically, CMP should be reserved to designate pathological lesion of the patellar articular cartilage; the diagnosis is appropriate when changes in cartilage can be directly visualized at arthroscopy. However, it is not always thought of as necessary to carry out an invasive procedure such as arthroscopy to diagnose an often benign and self-limiting condition [6]. In some instances, patients with histological changes of the patellar articular cartilage may be asymptomatic. But if the symptoms associated with CMP are severe enough to restrict daily activities, a combination of following conservative measures is indicated: quadriceps strengthening exercises, ice or heat compress, ultrasound, patellar mobilizations, and non-steroidal anti-inflammatory drugs (NSAID) [4]. Some patients become symptom-free with aforementioned treatments, but persistent severe symptoms associated with CMP would make surgical procedures necessary. Given that the patellofemoral joint is a complex articulation because of its dependence on both dynamic and static restrains for stability, the clinical effects of these invasive managements vary from series to series, and none of them has been universally successful [7]. More or less, the likelihood of postoperative return to vigorous physical activity is diminished.

NSAID are widely accepted to release retropatellar pain associated with CMP, but they cannot be taken for a long period due to various side-effects, and the analgesic effect of NSAID cannot be maintained once the medication is stopped. To date, complementary and alternative medicine is widely advocated to face the increasing demand for non-surgical and non-pharmacological approaches to treat CMP. As a mainstream of complementary and alternative medicine, acupuncture treatments based on Traditional Chinese Medicine (TCM) theory are commonly used to diminish patients' symptoms so that sport and other recreational pursuits can be resumed as quickly as possible. The safety and efficacy of acupuncture therapy can also assure a high level of patient compliance compared with conventional care. Unfortunately, there are no published systematic reviews summarizing the existing evidence as to clinical efficacy and safety of acupuncture therapy. Thus, the objective of the present study was to perform a systematic literature search of all published randomized controlled trials (RCT) and to compare the efficacy of acupuncture with that of NSAID in the treatment of CMP.

\section{Methods}

Literature Search Strategy

A systematic literature search of the databases CENTRAL, EMBASE, ISI Web of Science, and PubMed was conducted. Chinese databases including China Knowledge Resource Integrated Database (CNKI), Wan Fang Data, VIP, and Chinese Biomedical Literature database (CBM) were also scrutinized for the identification of trials. All the above databases were searched from their inception dates up to the latest issue (June 2016). No language restriction was used.

A combination of Medical Subject Headings (MeSH) and free terms was used to scrutinize all the potentially relevant articles. MeSH were slightly modified according to the specifications of each database. By way of example, the search strategy for PubMed was as follows: ('Chondromalacia Patellae'[Mesh] or Chondromalacia Patellae or patellar chondromalacia) and ('Acupuncture Therapy'[Mesh] or acupuncture or electroacupuncture or needle or meridian or acupoint). For Chinese databases, we used free text terms like 'Zhen (acupuncture)' or 'Jiu (moxibustion)' or 'Xue Wei (acupoint)' and 'Bin Gu Ruan Hua (chondromalacia patellae)'. In addition, the bibliographies of relevant systematic reviews and clinical guidelines were manually searched. We also searched the reference section of each study.

\section{Inclusion Criteria}

Studies that met the following criteria were included: 1) the enrolled patients were diagnosed with CMP; no restrictions on race, age, or sex were imposed; patients with CMP should be diagnosed by radiography, arthroscopy, or in accordance with the 'Standards for Diagnosis of Syndromes or Diseases of TCM and Evaluation of the Therapeutic Effect' issued by the State Administration of TCM in 1994 [8];2) the included studies were required to be RCT aiming to assess the efficacy of acupuncture therapy for CMP; 3) experimental groups mainly received acupuncture therapy (including, among other techniques, needles, moxibustion, acupressure, electroacupuncture, acupoint injection), either alone or in combination with rehabilitation training (such as quadriceps strengthening exercises), without differentiating between acupuncture techniques, acupoints, or needle materials; 4) patients in control groups were treated with NSAID, such as diclofenac sodium and celecoxib; no specific types of drugs were imposed; once the patients in the experimental groups accepted rehabilitation training, the same therapeutic methods had to be applied in control groups; 5) the outcome measures should include the clinical efficacy or pain scores. 


\section{Exclusion Criteria}

Four exclusion criteria for the identified studies were defined: 1) articles regarding animal experiments, reviews articles, case reports, or expert experience reports; 2) non-randomized studies; 3 ) studies that compared different acupuncture modalities or acupoints selection; 4) studies that were found to be duplicates in terms of retrieving or publishing.

\section{Data Extraction}

Two authors (Z.-T. Lv and Z.-Q. Li) screened each article independently and were blinded to the other reviewer's findings. The screening was performed in accordance with the predetermined inclusion and exclusion criteria to identify qualified articles. Data was extracted independently by the 2 reviewers from the articles they found to be eligible using a standardized collection form, which included first author, year of publication, study design, cohort sizes, baseline characteristics for participants in different groups, intervention treatments, main outcome assessments, follow-up periods post treatments, and adverse events. If the required information was not available in the included studies, attempts were made to contact the authors of the original papers via e-mail. Any disagreement between reviewers was resolved through discussion until a consensus was reached. The third reviewing author (A.-M. Chen) was consulted if a consensus could not be reached.

\section{Quality of the Studies}

The Cochrane Collaboration's tool was used to assess the risk of bias in the included studies, which based upon 7 items: random sequence generation, allocation concealment, blinding of participants and personnel, blinding of outcome assessment, incomplete outcome data, selective reporting, and other sources of bias [9]. The response for each criterion was reported as low risk of bias, high risk of bias, or unclear risk of bias. Two reviewers evaluated the quality of the trials independently.

\section{Data Synthesis and Analysis}

The meta-analysis and statistical analyses were performed using RevMan 5.3 analyses software of the Cochrane Collaboration. We extrapolated the risk ratio (RR) and the associated 95\% confidence interval (95\% CI) for treatment effect. The mean difference (MD) was calculated for pain scores using the same methodology. Before the data of the included studies was combined, heterogeneity between trial results was estimated using a standard chi-square test and the Higgins $\mathrm{I}^{2}$ test $\left(\mathrm{P}>0.1\right.$ and $\mathrm{I}^{2}<50 \%$ indicate acceptable heterogeneity). We pooled data across studies using random effect models if statistical heterogeneity existed; otherwise, a fixed effect model was used. In case of heterogeneity, a subgroup analysis was conducted. Publication bias was assessed via a funnel plot.

\section{Results}

\section{Literature Search Results}

An initial search of RCT yielded 406 literature citations, including 110 records from Wanfang, 118 from CNKI, 50 from VIP, 101 from CBM, 6 from PubMed, 6 from CENTRAL, 7 from EMBASE, and 8 from ISI Web of Science. 216 studies were deleted because they were duplicates. According to the predetermined selection criteria, 16 potentially relevant studies were selected and retrieved for a full-text assessment after screening titles and abstracts. Of these remaining 16 articles, 3 studies were excluded because they used complex therapy, 3 studies were excluded because they used unsuitable control intervention, and 3 studies were removed because they were duplicates for publica-

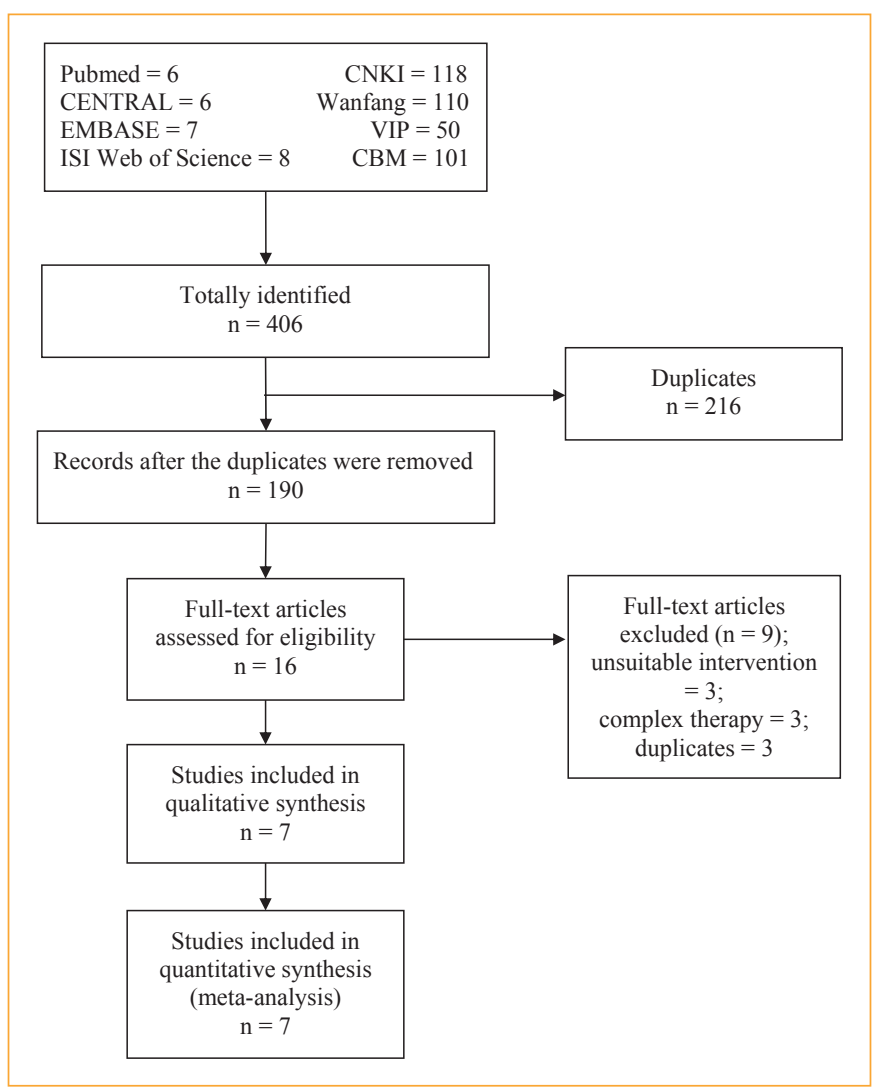

Fig. 1. Flowchart of the literature search and study selection.

tion. Finally, 7 studies met our inclusion criteria and were utilized for meta-analysis. The literature screening process is summarized in a flowchart in figure 1.

\section{Study Characteristics}

The main characteristics of the 7 trials are listed in table 1. All of these studies were conducted by Chinese investigators and published between 2006 and 2015. Each study was performed at a single center. The 7 RCT included a total of 707 patients with CMP: 364 patients in acupuncture groups and 343 patients in NSAID groups. The participants' age ranged from 20 to 79 years. All trials used 2-parallel-arm designs and acupuncture was compared with NSAID. In the acupuncture groups, EX-LE4 and EX-LE5 were the most commonly selected acupoints for acupuncture administration. The drugs administered to the NSAID groups included diclofenac sodium, meloxicam, and celecoxib; the duration of treatment ranged from 2-6 weeks. For the outcome measures, a clinical effect was observed in 5 studies [10-14]; visual analogue scale (VAS) scores were measured in 2 studies $[15,16]$.

\section{Quality Assessment}

The methodological quality of selected trials was assessed using the Cochrane Collaboration's tool. All of the studies included sug- 


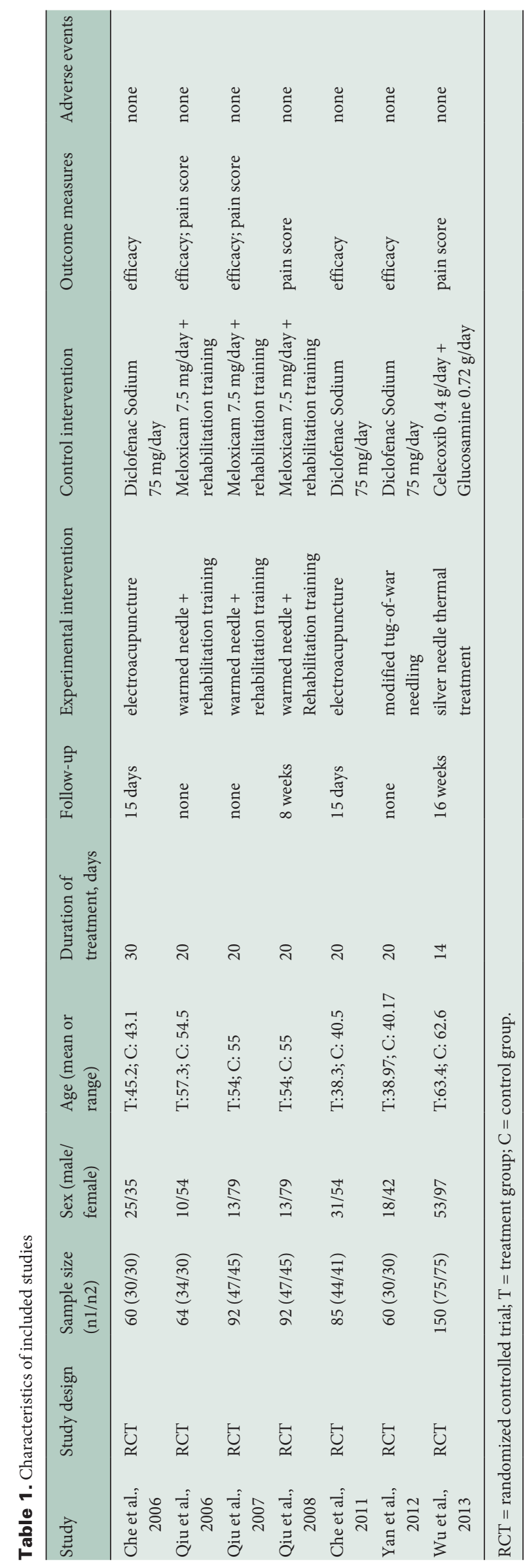

gested randomization, but only 2 studies $[11,16]$ reported the method of random sequences generation. No study provided information about allocation concealment. In our included studies, it was not feasible to blind patients and therapists; no study referred to blinding of outcome assessment. We considered that the outcome assessments were probably influenced by lack of blinding. Thus, the blinding of outcome measurement was judged to be at high risk for bias, and there was a high risk of bias regarding the blinding of participants or personnel in all studies. When it comes to selective reporting bias, there was a low risk since we only included studies which used clinical effect or pain scores as outcome measures. The baseline data of all studies showed similarity. Acceptable compliance seemed to have been obtained by all enrolled participants. In general, the methodological and reporting qualities of the selected studies were judged to be poor. The judgment of the risk of bias was presented by means of corresponding forest plot (fig. 2, 3).

\section{Meta-Analyses Results}

\section{Clinical Effect}

Five studies [10-14] involving 465 patients employed clinical effect as outcome measure; the therapeutic effect was evaluated in accordance with the 'Standards for Diagnosis of Syndromes or Diseases of TCM and Evaluation of the Therapeutic Effect' issued by the State Administration of TCM in 1994 [8]. Since there was no evidence for heterogeneity between studies $\left(\mathrm{Chi}^{2}=6.52\right.$; degrees of freedom $\left.(\mathrm{df})=4(\mathrm{P}=0.16) ; \mathrm{I}^{2}=39 \%\right)$, the fixed-effects model was utilized for statistical analysis. The pooled effects of 6 individual study results indicated that acupuncture therapy further improved clinical effect when compared with patients in NSAID groups $(\mathrm{RR}=2.57$; 95\% CI 2.02, 3.27; $\mathrm{P}<0.00001)$ (fig. 2).

\section{VAS Score}

Two studies $[15,16]$ involving 242 patients used pain scores as outcome measure. The pain degree is quantitated using a VAS in which a range from 0 to 10 points is used to describe pain degree: 0 points indicate no pain at all, and 10 points indicate the most severe pain that one can imagine. Data extracted from 2 studies showed obvious heterogeneity in the consistency of study results $\left(\mathrm{Chi}^{2}=6.58 ; \mathrm{df}=1(\mathrm{P}=0.01) ; \mathrm{I}^{2}=85 \%\right)$. Thus, the random effect model was employed for statistical analysis. The pooled effects of 2 independent trial results suggested that acupuncture therapy had further improved pain scores in patients with CMP when compared with NSAID treatment $(\mathrm{MD}=-1.49 ; 95 \% \mathrm{CI}-2.37,-0.62$; $\mathrm{P}<0.0008$ ) (fig. 3).

\section{Adverse Events}

None of the studies reported adverse events associated with acupuncture therapy. Only 1 study [11] reported security analysis. None of the patients seemed to have had unusual upset in treatment. Routine of blood, urine and stool, liver and renal functions were not abnormal after acupuncture treatment. 
Fig. 2. Forest plot of clinical effects. The pooled clinical effect, represented by the black diamond, demonstrates a significant difference favoring acupuncture therapy $(\mathrm{P}<0.00001)$.

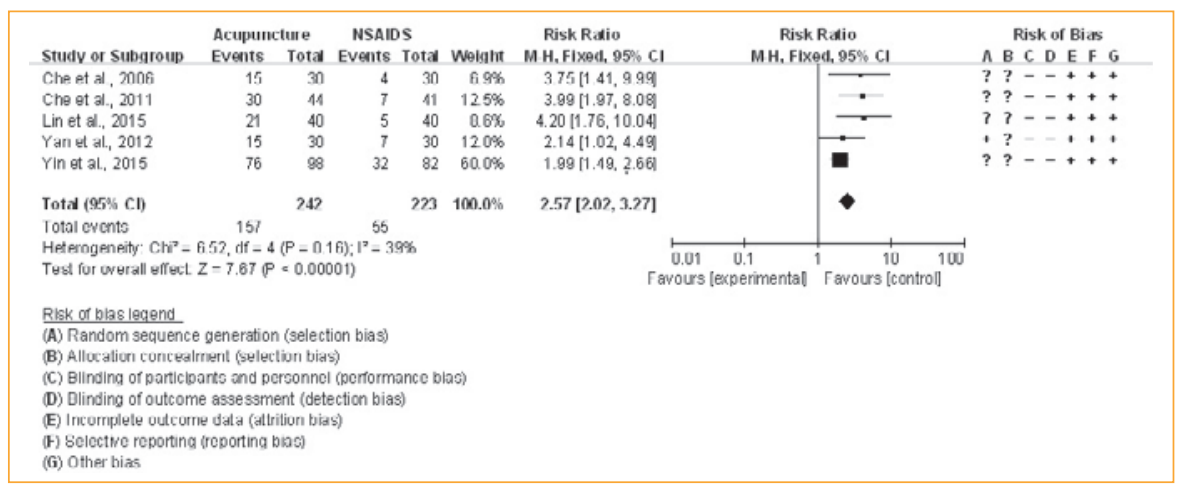

Fig. 3. Forest plot of pain scores. The pooled clinical effect, represented by the black diamond, demonstrates a significant difference favoring acupuncture therapy $(\mathrm{P}<0.00001)$.

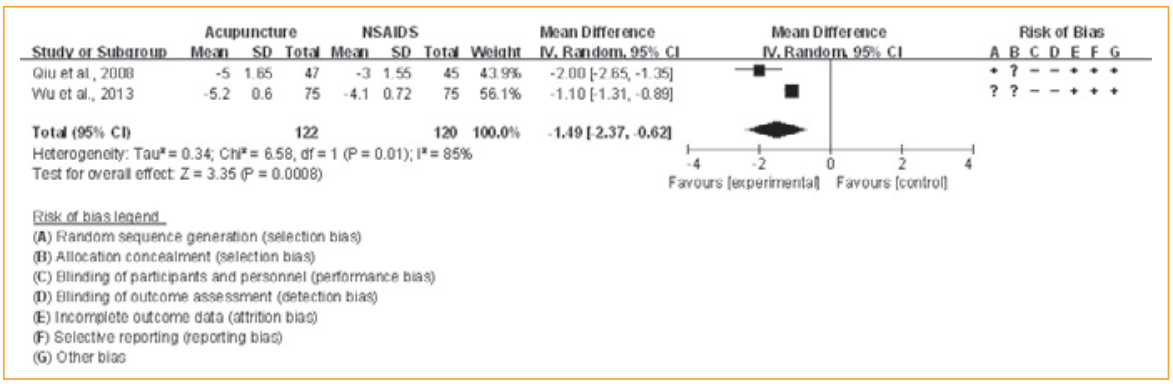

\section{Publication Bias}

Publication bias was explored by funnel plot (fig. 4). Points in figure 4 present asymmetry, suggesting an obvious publication bias.

\section{Discussion}

\section{Summary of Evidence}

To our knowledge, this is the first meta-analysis of acupuncture therapy in the treatment of CMP. Seven RCT involving 707 patients with CMP were selected for this meta-analysis. Based on the findings of the present systematic review, acupuncture therapy can further improve the clinical effect and pain scores when compared with NSAID controls. However, the potential beneficial effect of acupuncture is possibly overstated owing to the low general methodological quality of the included RCT. Conclusions regarding the safety of acupuncture therapy cannot be drawn since the eligible trials provide no evidence on this aspect.

\section{Mechanism of Acupuncture Therapy}

CMP is of great interest because of the high incidence of disabling pain in patients who are often young and athletic. It is one of the most common syndromes involved in anterior knee pain [17]. Harris et al. [18] divided the patients into 4 etiological groups: the maltracking patellae, the unstable patellae, posttraumatic chondromalacia, and idiopathic CMP. This condition, which presents with clinical symptoms, normally has an insidious onset. The process of

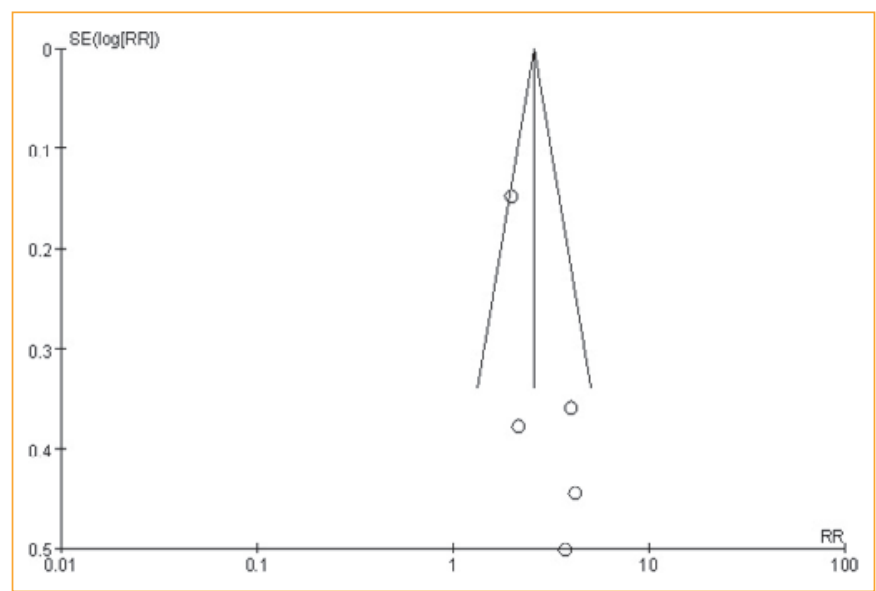

Fig. 4. Funnel plot of acupuncture versus NSAID: clinical effect.

CMP is deemed to be caused by a trauma to superficial chondrocytes leading to a proteolytic enzymic breakdown of the matrix. Since the clinical manifestations associated with CMP mostly occur before gross breakdown of articular cartilage, successful implementation of conservative management at this point may result in control of further cartilage breakdown.

Generally, the conservative therapy is based on rehabilitation training of the vastus medialis muscles, such as straight leg raise and semi-squat. This specific kinetic therapy improves the patellar alignment by modifying strength of muscles and harmonization of actions. After muscular rehabilitation protocols, the proprioceptive ability is also significantly increased. Furthermore, the rehabilitation training process prevents unequal pressure on the patellofemoral joint surface and helps to reduce joint inflammation and to 
heal the syndrome by correcting the pattern of load distribution on the joint surface [19]. And the muscle strengthening exercise itself has a positive influence on articular nutrition via compression of the articular surfaces and decreased edema [20].

The clinical problem of chronic knee pain encompasses a wide group of causes, for e.g., osteoarthritis of knee, rheumatoid arthritis, and CMP. The ideal pain relieving effect in osteoarthritis of knee and rheumatoid arthritis has been proven by well-established systematic reviews $[21,22]$. Acupuncture stimulation of the skin and muscles induces a variety of somatomotor and autonomic responses; it acts by coordinating different groups of afferent fibers, thus leading to pain relief [23]. Electroacupuncture blocks pain by activating a variety of bioactive mechanisms including opioids, which desensitize peripheral nociceptors and reduce proinflammatory cytokines peripherally and in the spinal cord as well as serotonin and norepinephrine [24]. With acupuncture stimulation, levels of endogenous opioids are increased in the plasma [25]. Plasma levels of beta-endorphin, an endogenous opioid analgesic, are often reported as they relate to acute and chronic pain outcomes [26]. From a macro viewpoint, acupuncture therapy helps to promote blood circulation of the knee joint and joint fluid circulation in the articular cavity and to strengthen the nutrition of articular cartilage. Additionally, neuromuscular electric stimulation increases muscle strength and interferes with knee instability [27]. According to the TCM theory, CMP belongs to the 'Bi syndrome'; the pathogenesis of CMP mainly refers to muscle strain on the basis of deficient 'Right Qi'. The most commonly selected acupoints in our included studies were EX-LE4 and EX-LE5. Applying warm needling to the above-mentioned acupoints helps to strengthen muscles and bones, warm channels, disperse cold, clear channels, stop pain, promote blood circulation, and remove blood stasis. Intensive silver needling has been reported to be effective in reducing local inflammatory reaction [28].

\section{Study Limitations}

The overall methodological quality of our selected trials was judged to be poor, which might reduce the validity and value of the evidence examined in the present review. Many studies failed to describe their experimental methodological design in detail. All of the included trials claimed randomization, but most of them did not provide information about randomization sequence generation to estimate whether the randomization procedures had been carried out appropriately. None of the studies employed the strategy of double-blinding, which might unsheathe placebo effects and exaggeration of conclusions. All studies failed to provide information about allocation concealment. Therefore, implementation of allocation concealment could not be assessed. Three studies did not report follow-ups post treatment; here, the long-lasting beneficial effect of acupuncture could not be observed adequately. Cost effectiveness and quality of life are distinct advantages of acupuncture therapy. Unfortunately, no study reported information on this aspect.
In this study, the specific interventions employed in the eligible trials included electroacupuncture, warm needle, silver needle, and modified tug-of-war needling. These techniques were considered as one type of therapy, without differentiating acupoint selection or acupuncture forms. Consequently, the findings of this review might indicate overall efficacy trends, but definitive conclusions could not be drawn due to the limited evidence provided by the included studies. To further assess the beneficial effect of acupuncture therapy in CMP, large-scale RCT with rigorous study design are required. Our findings in this review suggest that acupuncture alone or in combination with rehabilitation training might be an option for conservative treatment to release pain and other symptoms associated with CMP before invasive surgeries.

\section{Suggestion for Future Research}

The included studies comprised various methodological deficiencies, and the findings of the present systematic review are somewhat limited by this low overall methodological quality. Future RCT on acupuncture therapy should employ improved methodologies and reporting specifications as follows: 1) all clinical acupuncture studies should be registered and comply with the 'revised standards for reporting interventions in clinical trials of acupuncture' [29]; 2) future studies should report detailed information about randomization sequences generation and allocation concealment; 3) studies on acupuncture therapy should be blinded and placebo controlled if possible; 4) the number of dropouts and reasons for withdrawal should be presented in a flowchart, and intention-to-treat analysis should be used to assess the clinical effect; 5) the standard of diagnosis should be unified worldwide, and at least 6 months of follow-up should be required; 6) adverse events associated with acupuncture should be reported in detail, cost effectiveness should be noteworthy in future studies; 7) given that most studies on acupuncture are performed in China, clinical trials within a Western context are encouraged.

\section{Conclusion}

In summary, the results of the present systematic review suggest that acupuncture therapy can further improve clinical effects and pain scores in patients with CMP when compared with NSAID controls. However, the conclusion should be interpreted cautiously due to the poor methodological quality of the included studies. No adverse events were documented in these trials. Additional RCT with large-scale, rigorous study design and strict reporting specifications are required.

\section{Acknowledgement}

This study was funded by grants from the National Natural Science Foundation of China (No. 81472082). 


\section{Contribution}

An-Min Chen developed the idea to this analysis and prepared the final version of this paper. Zheng-Tao Lv and Zhi-Qiang Li did the literature search and evaluated the data. Xiang Zhou, Wen-Wen Ma and Jin-Ming Zhang critically revised this manuscript.

\section{Disclosure Statement}

The authors declare that there are no competing interests concerning this study.

\section{References}

1 Bentley G, Leslie IJ, Fischer D: Effect of aspirin treatment on chondromalacia patellae. Ann Rheum Dis 1981;40:37-41.

2 Bentley G: The surgical treatment of chondromalacia patellae. J Bone Joint Surg Br 1978;60:74-81.

3 Macmull S, Jaiswal PK, Bentley G, Skinner JA, Carrington RW, Briggs TW: The role of autologous chondrocyte implantation in the treatment of symptomatic chondromalacia patellae. International Orthop 2012; 36:1371-1377.

4 McConnell J: The management of chondromalacia patellae: a long term solution. Aust J Physiother 1986;32: 215-223.

5 Bentley G: Articular cartilage changes in chondromalacia patellae. J Bone Joint Surg Br 1985;67:769-774.

6 Murphy E, FitzGerald O, Saxne T, Bresnihan B: In creased serum cartilage oligomeric matrix protein levels and decreased patellar bone mineral density in patients with chondromalacia patellae. Ann Rheum Dis 2002;61:981-985.

7 Yildiz Y, Aydin T, Sekir U, Cetin C, Ors F, Alp Kalyon $\mathrm{T}$ : Relation between isokinetic muscle strength and functional capacity in recreational athletes with chondromalacia patellae. Br J Sports Med 2003;37:475-479.

8 State Administration of Traditional Chinese Medicine: Standards for Diagnosis of Syndromes or Diseases of TCM and Evaluation of the Therapeutic Effect. Nanjing, Nanjing University Press, 1994.

9 Higgins JPT, Green S (eds): Cochrane Handbook for Systematic Reviews of Interventions Version 5.1.0 (updated March 2011). The Cochrane Collaboration, 2011; http://handbook.cochrane.org.

10 Yin CF, Er ZJ, Lv SZ: Clinical observation of electroacupuncture in the treatment of chondromalacia patellae. J Med Theory Pract 2015;28:2651-2652.
11 Yan J, Jiang SY, Meng J, Huang JL, Zheng B, Liang NY: Clinical observation on chondromalacia patellae treated with modified tug of war needling method. Chin Arch Tradit Chin Med 2012;30:1111-1112.

12 Lin HM, Huang L, Yang FY: Clinical research on treating chondromalacia patellae by thermal moxibustion plus massage. Clin J Chin Med 2015;7:47-49.

13 Che T, Qiu ML, Xin ZP, Shao P: The electric needle treatment patella softens clinical research of the disease. Chin J Pract Chin Mod Med 2006;19:791-792.

14 Che T, Qiu ML, Sun J, Li YF: Clinical research of treating chondromalacia patellae by ea patellofemoral. Chin J Pract Chin Mod Med 2011;24:20-21.

15 Wu HS, Li CX, Cheng XZ, Wu JL: Efficacy of intensive silver needle thermal treatment for the treatment of knee pain caused by chondromalacia patellae. Pain Clin J 2013;9:267-269.

16 Qiu L, Zhang J, Zhang M, Zheng X, Xie F, Gao LN, Chen DW: Clinical effect of treating the pain from chondromalacia patellae with acupuncture with warmed needle combined with rehabilitation practise. J Sichuan Trad Chin Med 2008;26:103-105.

17 Casscells SW: Chondromalacia of the patella. J Pediatr Orthop 1982;2:560-564.

18 Ogilvie-Harris DJ, Jackson RW: The arthroscopic treatment of chondromalacia patellae. J Bone Joint Surg Br 1984;66:660-665.

19 Bakhtiary AH, Fatemi E: Open versus closed kinetic chain exercises for patellar chondromalacia. Br J Sports Med 2008;42:99-102; discussion 102.

20 Irrgang JJ, Pezzullo D: Rehabilitation following surgical procedures to address articular cartilage lesions in the knee. J Orthop Sports Phys Ther 1998;28:232-240.
21 Corbett MS, Rice SJ, Madurasinghe V, Slack R, Fayter DA, Harden M, Sutton AJ, Macpherson H, Woolacott NF: Acupuncture and other physical treatments for the relief of pain due to osteoarthritis of the knee: network meta-analysis. Osteoarthritis Cartilage 2013;21:12901298.

22 Wang C, de Pablo P, Chen X, Schmid C, McAlindon T: Acupuncture for pain relief in patients with rheumatoid arthritis: a systematic review. Arthritis Rheum 2008;59:1249-1256.

23 Kagitani F, Uchida S, Hotta H: Afferent nerve fibers and acupuncture. Auton Neurosci 2010;157:2-8.

24 Zhang R, Lao L, Ren K, Berman BM: Mechanisms of acupuncture-electroacupuncture on persistent pain. Anesthesiology 2014;120:482-503.

25 Lv ZT, Song W, Wu J, Yang J, Wang T, Wu CH, Gao F, Yuan XC, Liu JH, Li M: Efficacy of acupuncture in children with nocturnal enuresis: a systematic review and meta-analysis of randomized controlled trials. Evid Based Complement Alternat Med 2015;2015:320701.

26 Bruehl S, Burns JW, Chung OY, Chont M: What do plasma beta-endorphin levels reveal about endogenous opioid analgesic function? Eur J Pain 2012;16:370-380.

27 Dos Santos RL, Souza ML, Dos Santos FA: Neuromuscular electric stimulation in patellofemoral dysfunction: literature review. Acta Ortop Bras 2013;21:52-58.

28 Feng CY, Chen H, Wang FG, Gao Q, Wang J, Hou JS: Effect of warmed silver needle therapy on the level of interleukin-8 in skeletal muscle of rabbit with chronic quadriceps injury. Chin J Clin Rehab 2005;9:98-99.

29 MacPherson H, Altman DG, Hammerschlag R, Youping L, Taixiang W, White A, Moher D, Group SR: Revised STandards for Reporting Interventions in Clinical Trials of Acupuncture (STRICTA): extending the CONSORT statement. PLoS Med 2010;7:e1000261. 